\title{
Orthodontic intervention of an impacted upper left central incisor due to odontoma. A case report
}

\author{
Mwakatobe A $\mathbf{J}^{1}$, Kahabuka FK ${ }^{2}$
}

1. School of Assistant Dental Officers (ADO). Muhimbili National Hospital. Dar es Salaam, Tanzania.

2. Department of Preventive and Community Dentistry. School of Dentistry, Dar es Salaam, Tanzania.

Mwakatobe A J,. Kahabuka FK. Orthodontic intervention of an impacted upper left central incisor due to odontoma. A case report. Tanz Dent J 2008; 15 (1): 17 -21

\begin{abstract}
:
Aim: To present a case of a compound odontoma impeding eruption of the permanent upper left central incisor and to narrate its management. Case history: A 12-year-old girl attended at Muhimbili National Hospital dental clinic complaining of a missing upper anterior tooth. At the age of 3 years the patient sustained injury on the anterior part of the upper jaw. A decidous tooth was fractured and eventually extracted. Oral tissues were healthy and the dentition was caries free. Molar relationship on the right-half a unit was class II, on the left full unit class II. Moderate crowding was observed on the upper right and lower right arches, whereas, mild crowding was seen on the lower left arch. No detrimental para functional habits were noted. Radiographic examination revealed the presence of a radiopaque mass with features consistent with an Odontoma, it was overlying an impacted upper left central incisor and had a size of about 4 X $4 \mathrm{~mm}$. Treatment done: The odontoma was surgically removed. A fixed orthodontic appliance was used to facilitate traction and correction of malalignement of the impacted upper left central incisor.Treatment outcome: Successful removal of the odontoma, full exposure of the crown of upper left central incisor and good alignment on the arch were achieved. Crowding on the upper and lower arches was relieved and leveling of both arches together with improvement of midline shift resulted in good facial aesthetics. Conclusion: Successful removal of the impeding odontoma followed by orthodontic management of an impacted incisor with a fixed appliance successfully restored the child's aesthetics and self esteem.
\end{abstract}

Correspondence: Mwakatobe AJ, School of Assistant Dental Officer (ADO), Muhimbili National Hospital, Dar es Salaam, Tanzania: E-mail: ambege@ yahoo.com

\section{Introduction}

Odontomas are not true neoplasms but are malformations of dental hard tissues (1). They are classified as compound when they appear as multiple small "teeth" consisting of identifiable enamel and dentine usually within a fibrous sac, and complex when they are in form of a congealed irregular mass of the dental hard tissues. Compound odontomas have been reported to have a mean age of occurrence of 14.8 years compared to 20.3 years of age for complex odontomas (2).

Literature reveals that approximately $10 \%$ of all odontogenic tumors of the jaws are compound odontomas (3). Furthermore, Palser (1993) reported that, compound odontomas occur almost exclusively in the anterior segment of the jaws and are found more often in the maxilla than in the mandible. On the other hand, complex odontomas are most commonly observed in the region of the mandibular third molars in males and in the maxillary tuberosity region in females (4). Most odontomas occurring in the anterior segment of the jaws are compound composite $(61 \%)$, whereas the majority (34\%) in the posterior segment are complex composite (3).

Radiographically, a compound odontoma appears as a collection of tooth like-structures of varying size and shape surrounded by a narrow radiolucent zone. The complex odontoma appears as a calcified mass with a radio density of tooth structure, which is also surrounded by narrow radiolucent rim (4).

Generally, odontomas are asymptomatic, although occasionally signs and symtopms relating to their presence do occur. An odontoma has a limited growth potential, however, it is usually removed because it may predispose to cystic change, interfere with eruption of permanent teeth and sometimes may cause considerable bone destruction. An odontoma may displace teeth, creating diastema; it may also impende normal teeth from erupting $(5,6)$. Shafer et al. (6) reported that generally the symptoms consisted of unerupted or impacted teeth, retained decidous teeth, swellings and occasionally of infection. The etiology of the odontoma is unknown, but it has been suggested that local trauma or infection may lead to the occurence of such a lesion. Studies have shown that odontomas can be treated successfully by conservative surgical enucleation with little possibility of recurrence $(2,3)$.

\section{Case History}

A 12-years-old girl reported at a dental clinic Muhimbili National Hospital complaining of an unerupted permanent upper tooth while a similar tooth on the contralateral side had erupted (Figure 1). Initially, the child's mother thought it was just a normal delay, but as time elapsed, she thought of seeking dental attention. Dental history revealed 
that, at the age of 3 years the patient sustained trauma on the anterior part of upper jaw after falling down while playing with her mates. A deciduous tooth was fractured and eventually extracted at a local clinic. Furthermore, the mother reported that one upper left posterior tooth was removed a year prior to their visit to our clinic due to improper eruption.

\section{Examination}

On examination the child was generally healthy looking; she had no allergies or any apparent medical problem. There were no significant medical conditions contraindicating orthodontic treatment.

On extra-oral examination, the child had a class I skeletal pattern. This was observed by viewing the soft tissue facial profile, and by palpating the alveolar bases over the apices of the upper and lower incisors in the midline. The Frankfortmandibular planes angle (FMPA) was average, both lines intersected at the back of the skull occiput. Her lips were competent. No facial asymmetry, no abnormal temporal mandibular joint signs or symptoms were detected.

On intra-oral examination; oral hygiene was fair, oral soft tissues were healthy and the dentition was caries free. No detrimental para functional habits were prominent. Molar relationship on the right was half a unit class II, on the left was full unit class II. Moderate crowding was observed on the upper right and lower arch, whereas mild crowding was seen on the lower left arch. The lower second premolars were lingually blocked and rotated mesio-lingually. The upper right first premolar was palatally blocked and rotated mesio-palatally. The upper left canine was labially erupted, infraoccluded and distally inclined. The upper right central incisor had attrition while the upper left central incisor and the upper right first premolar were missing in the oral cavity. There was an upper centerline shift to the left ( $\sim 2 \mathrm{~mm})$, lower centerline shift to the right $(\sim 1 \mathrm{~mm})$.

\section{Investigations}

For orthodontic records, an orthopantomogram, a standard occlusal radiograph, photographs and a study model were made. A lateral cephalometric radiograph could not be obtained due to technical reasons.

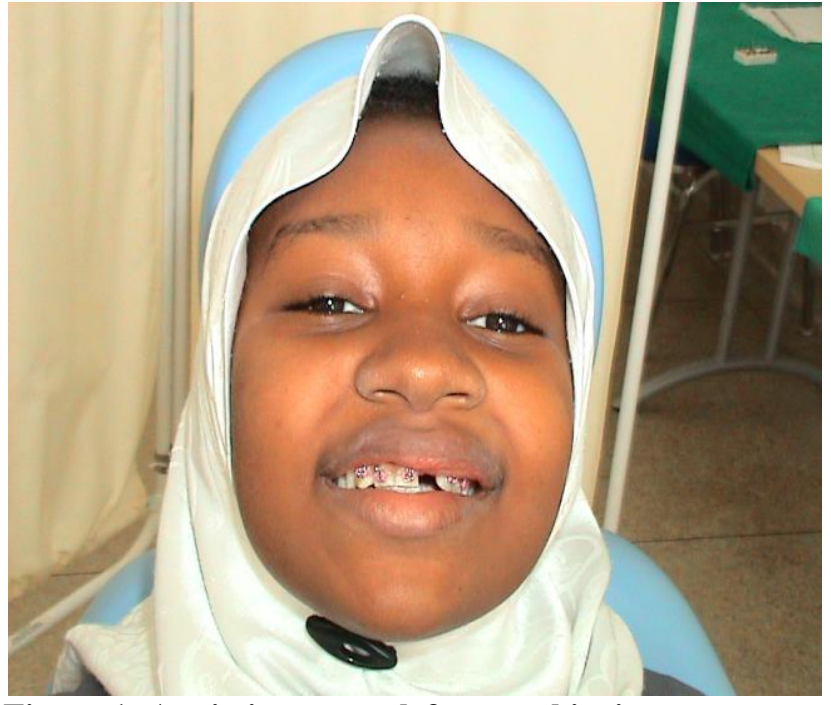

Figure 1: A missing upper left central incisor

\section{Orthopantomogram (OPG) interpretation}

All permanent teeth were confirmed to be present on the OPG except the upper left first premolar. A radiopaque mass of about $4 \times 4 \mathrm{~mm}$ with features consistent with an odontoma was seen overlying the impacted upper left central incisor . All third molars were at the early stage of development; their crowns were yet to calcify. Potential impactions were noted on both lower third molars, (Figure 2).

\section{Diagnosis}

The child's occlusion was classified as a class II division I Malocclusion on class I skeletal pattern with average FMPA. The upper left central incisor was impacted, the upper left canine was labially malpositioned, infraoccluded and distally inclined. There was moderate crowding on the right upper and lower arch and mild crowding on the left lower arch. Also observed was upper centerline shift to the left $(\sim 2 \mathrm{~mm})$; lower centerline shift to the right $(\sim 1 \mathrm{~mm})$. Molar relationship on the right was half a unit class II, on the left was full unit class II and incisor relationship was class I.

Regarding the overlying odontoma, histopathological investigation to confirm the diagnosis could not be done due to technical reasons. However, dental history, macroscopic and radiographic examination suggested a provisional diagnosis of a compound odontoma.

The Index of Orthodontic Treatment Need: Dental Health Component (IOTN DHC) scored 5i (very great), due to impended eruption of upper left central incisor caused by the overlying odontoma as suggested by Heasman (2003). 


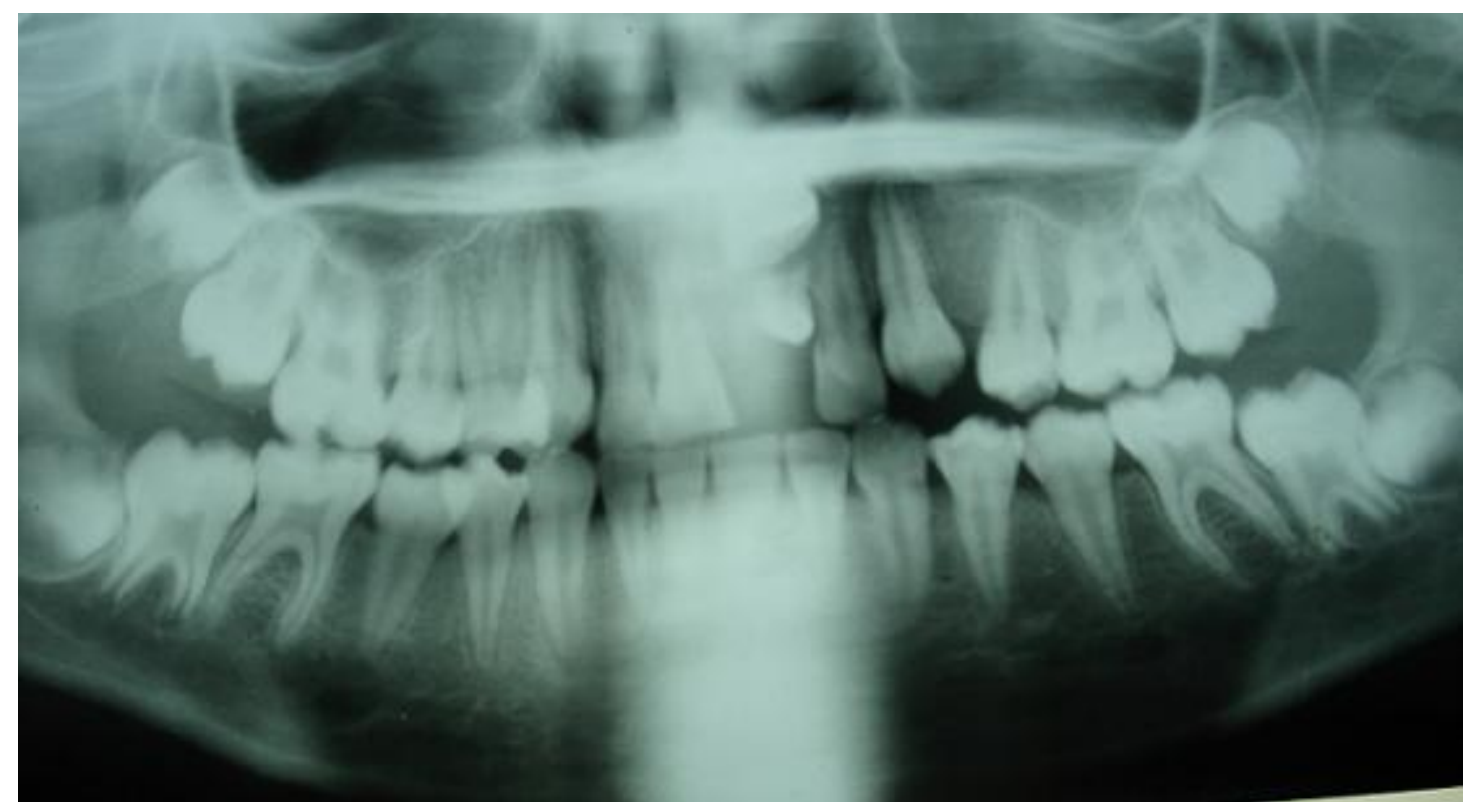

Figure 2: Odontomes impeding the crown of the upper left central incisor

\section{Treatment plan}

Oral hygiene instructions.

Dietary advice.

Fixed Orthodontic appliance on the upper and lower arch.

Removal of odontoma.

Attach an orthodontic eyelet to the upper left central incisor.

Traction of the impacted upper left central incisor. Alignment and leveling

Observe the upper arch response and review treatment accordingly.

Correction of molar, incisor and canine relationships.

Space closure.

Retention of the upper arch with bonded retainer to ensure long-term stability especially for the upper left central incisor.

Evaluate the position of lower third molars after treatment.

Management of the attrition on 11 with composite material.

Labial gingivoplasty may be required at a later stage in relation to upper left central incisor to obtain coincidence of the gingival margins on upper central incisors.

Routine follow up to the age of 18 years or more.

\section{Treatment outcome}

Largely the treatment objectives were achieved (figure 3 and figure 4) namely;

The Odontoma was removed and the crown of upper left central incisor was fully exposed and well aligned on the arch.
Crowding on the upper and lower arch was relieved.

Alignment and leveling of the upper and lower arches was achieved.

Improvement of midline shift was attained.

Improvement of facial aesthetics are accomplished.

The patient had good progress. At present the patient is in the final stages of treatment with preformed arch wire $(017 \times 025 \mathrm{SS})$ for torque control, space closure and final midline correction. After settling stage, canine to canine lingual bonded retainer will be placed in the upper arch for retention.

\section{Discussion}

Skeletal relationship was not analyzed radiographically because a lateral cephalometric radiograph could not be done due to unavailability of the service at the hospital. However, clinically, the child's profile had a class I skeletal pattern. This was observed by viewing the soft tissue facial profile, and by palpating the alveolar bases over the apices of the upper and lower incisors in the midline. The Frankfort-mandibular planes angle (FMPA) was average, both lines intersected at the back of the skull occiput. 


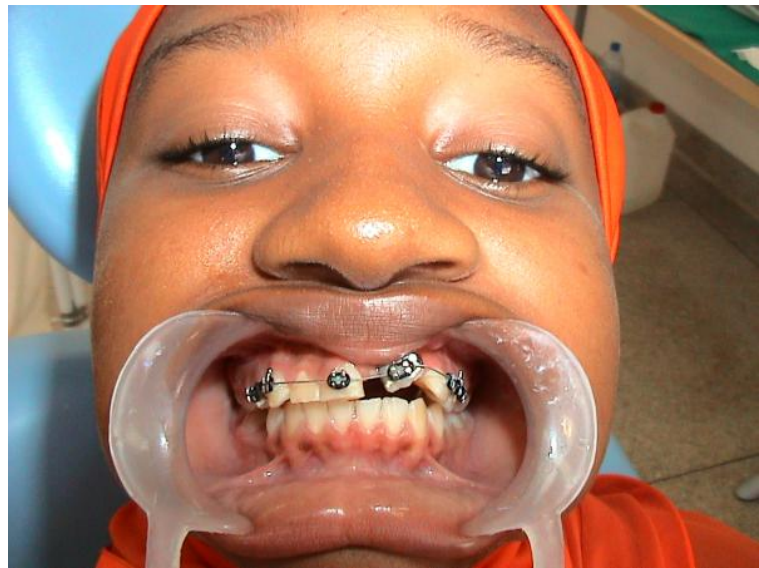

Figure 3: Two thirds of the crown the upper left central incisor exposed

The young girl was a very motivated patient with adequate parental support. Except for few missed appointments she was very cooperative throughout treatment. After surgical removal of the odontoma, a fixed appliance was selected to correct the malalignment and to facilitate traction of the impacted upper left central incisor. This was done in accordance to a well established fact that fixed orthodontic appliances can efficiently help to manage several problems at a time $(1,7)$. To ensure improved facial esthetics, the option of fixed appliance also guaranteed proper alignment and leveling of the upper and lower arch, correction of the mid line and correction of incisor and canine relationship.

The main challenge encountered was midline adjustment and correction of molar relationship; this was mainly due to the extracted upper left first premolar.

Class II molar relationship was a result of malalignment on both buccal segments of the upper arch, aggravated by a space on the impacted upper left central incisor and later by the extraction of upper left first premolar; which was a reason also for the midline shift to the left on the upper arch. The slight shift of midline to the right on the lower arch was most likely due to the lingually blocked lower right second premolar and a mild crowding on the lower left arch.

Parents reported a history of trauma when the girl was three years old. The trauma might have contributed to the occurrence of odontomas in this patient (1). For this reason, monitoring of patients who sustain injury at young age is thus underscored.

In the present case, Orthodontic records analyzed indicated that the treatment had minimal effect on the patient's soft tissue profile. Most of the treatment objectives were achieved. The patient and parents are very pleased with the results achieved so far.
It is obvious that fixed orthodontic appliances can efficiently help to manage several problems at a time. As reported by Mwakatobe (8), fixed orthodontic management poses a big challenge in Tanzania. Basic and essential instruments as well as materials necessary for fixed orthodontic appliances are very expensive and their availability is not guaranteed. Furthermore, there is minimal expertise on fixed orthodontic appliance among dentists in the country.

Odontomas are painless thus diagnosis is usually delayed and often is accidental when investigating for other complaints. Consequently, once suspected, accurate diagnosis is emphasized so as to make appropriate plan of management. The earlier the problem is discovered the higher are the chances of successful management. Even the patient being hereby reported visited the dental clinic complaining of a missing tooth. Upon investigation, odontomas were revealed.

\section{Conclusion}

Successful removal of the impeding odontoma followed by orthodontic management of an impacted incisor with a fixed appliance could successfully restore the child's aesthetics and self esteem.

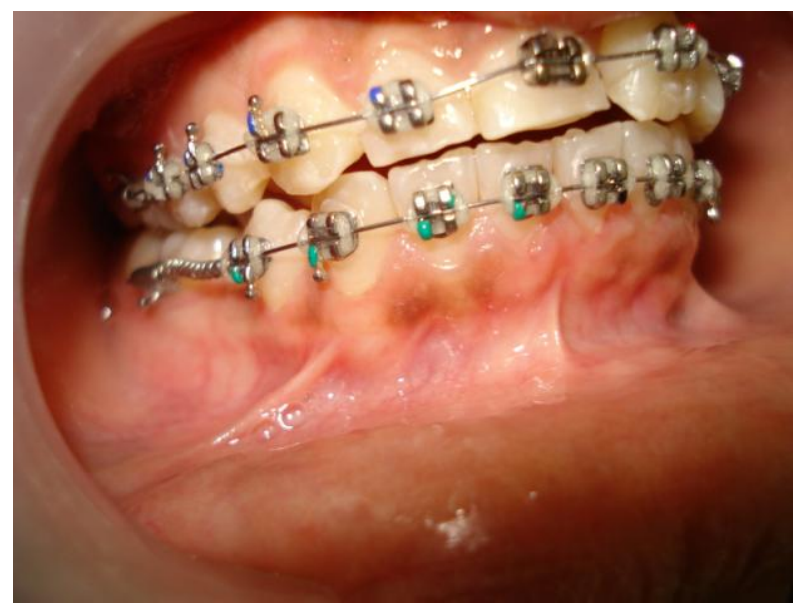

Figure 4: Crown of the upper left central incisor fully exposed and well aligned on the arch

\section{Recommendation}

We recommend multidisciplinary approach in managing similar cases. Patients are likely to benefit more if paediatric dentists, orthodontists, oral surgeons and oral pathologists will work together to make accurate diagnosis and management plan. 


\section{Acknowledgements}

We thank the child for cooperation and her mother for giving consent to use the findings and photographs in this publication.

\section{References}

1 Haesman P. MASTER DENTISTRY. Restorative Dentistry, Paediatric Dentistry and Orthodontics. Vol.2. Churchill Livingstone. 2003. P 202, 234, 238, 304-306.

2 McDonald R E., Avery D R., Dean J A. Dentistry for the Child and Adolescent. Eighth Edition. Mosby 2004. P 164 - 165.

3 Singh S. Singh M. Singh ., Khandelwal D. Compound composite odontome associated with an unerupted deciduous incisor - a rarity. J Indian Soc Pedod Prev Dent 2005;146-50.
4. Pasler F. A. Color Atlas of Dental Medicine. Radiology. 1993 Thieme. New York. P199 212.

5. Zoremchhingi; Joseph T; Varma B; Mungara J. A compound composite odontoma associated with unerupted permanent incisor-a case report. J Indian Soc Pedod Prev Dent. 2004; 22(3):114-7.

6. Shafer W.G., Hine M.K. \& Levy B.M. A textbook of Oral pathology Fourth Ed W.B. Saunders \& company, Philadelphia 1999.

7. Profit W.R, Fields H.W, Sarver D.M. Contemporary Orthodontics. Fourth Edition. Mosby 2007.P 407-429.

8 Mwakatobe A. J. Fixed orthodontic appliances. An update. Tanzania Dental Journal Vol.13 No. 2, November 2006.P 64 - 68 .

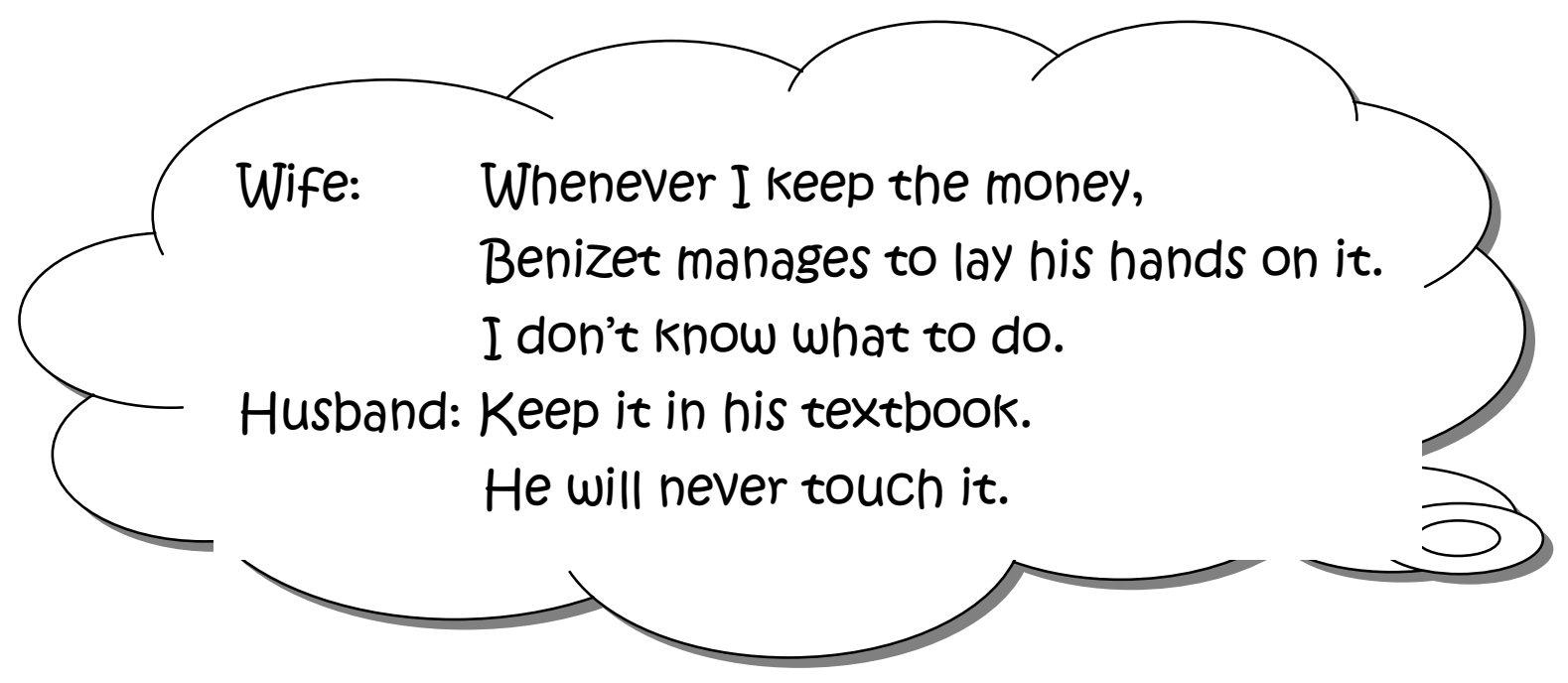

Teacher: Why are you late?

Wilson: Because of the sign.

Teacher: What sign?

Wilson: The one that says "School ahead go slow" that is what I did. 